\title{
Téléphone mobile et santé mobile : une approche info- communicationnelle du corps comme objet connecté
}

\author{
Mobile Phone and Mobile Health: Information and Communication of the \\ Body as a Connected Object
}

\author{
Brigitte Juanals $^{1}$ \\ ${ }^{1}$ Laboratoire EA 4262 IRSIC, Aix-Marseille Université, France, brigitte.JUANALS@univ-amu.fr
}

\begin{abstract}
RÉSUMÉ. Cette communication traite des enjeux sociopolitiques et des modes d'accès mobile à l'information en ligne à partir d'un terminal informatique nomade, en centrant l'attention sur les téléphones mobiles. Les enjeux de l'Internet des objets sont étudiés dans les usages et les systèmes techniques en santé mobile qui ont déstabilisé l'organisation traditionnelle de la santé. Nous analysons les modalités selon lesquelles de nouvelles formes éditoriales, centrées sur les nouveaux modes de production et de diffusion d'informations, conditionnent l'accès mobile à des contenus. Nous montrons que ces modes d'accès sont le fruit des relations croisées entre des innovations techniques, les modèles socioéconomiques des acteurs industriels de la téléphonie mobile, les pratiques éditoriales des éditeurs d'applications et les modes de régulation de ce domaine intersectoriel.

ABSTRACT. This paper deals with sociopolitical issues and mobile access to online information from a mobile computer terminal, putting the emphasis on mobile phones. The stakes of the Internet of things are studied in the uses and the technical systems in mobile health that have destabilized the traditional health organization. We analyze the ways in which new editorial forms, centered on the new modes of production and dissemination of information, provide mobile access to contents. We show that these modes of access are the result of the cross-relationships between technical innovations, socio-economic models of the industrial stakeholders in mobile telephony, the publishing practices of application publishers and the modes of regulation of this cross-sector area.

MOTS-CLÉS. santé mobile, accès mobile, smartphone, industries de la communication, médiation socio-technique, pratiques.

KEYWORDS. mobile health, mobile access, smartphone, communication industries, socio-technical mediation, practises.
\end{abstract}

\section{Introduction}

L'Internet des objets (IdO) étend le réseau Internet à des objets et à des lieux de l'environnement physique. Il est à l'origine d'un accroissement considérable des quantités de données issues des nouveaux usages qui y sont associés, contribuant ainsi à alimenter les données massives. Une problématique située au croisement des dimensions techniques et des usages sociaux le définit comme " un réseau de réseaux qui permet, via des systèmes d'identification électronique normalisés et unifiés, et des dispositifs mobiles sans fil, d'identifier directement et sans ambiguïté des entités numériques et des objets physiques et ainsi de pouvoir récupérer, stocker, transférer et traiter, sans discontinuité entre les mondes physiques et virtuels, les données s'y rattachant» [BEN 09: 15-23]. Dans cette communication, nous centrons l'attention sur les enjeux sociaux, politiques et économiques de l'IdO ; ils résident dans les modes d'accès décuplés à l'information caractéristiques de "l'informatique ubiquitaire » [GRE 06], dans la création de nouveaux services et dans l'exploitation des données conduite au moyen d'algorithmes. Dans ses usages sociaux, l'IdO s'incarne dans une grande diversité d'objets connectés. Il fait apparaître la complexité des technologies de l'information et de la communication dans leurs dimensions d'interopérabilité et de préservation de la sécurité et de la fiabilité des données. Les traitements réalisés par des acteurs publics ou privés posent les questions de la traçabilité de l'humain dans les systèmes d'information et de la protection des données personnelles des usagers de ces dispositifs sociotechniques [JUA 09, 14]. 
Inscrite dans le champ des Sciences de l'information et de la communication, cette communication traite des enjeux sociopolitiques et des modes d'accès mobile à l'information englobant la production et l'accès à des contenus et à des services en ligne à partir d'un terminal informatique nomade, en centrant l'attention sur les téléphones mobiles (smartphones). Les enjeux de l'IdO sont abordés dans les usages et les systèmes techniques en santé mobile (mobile health) qui ont déstabilisé l'organisation traditionnelle de la santé.

Nous présenterons en premier lieu les spécificités du domaine hybride de la santé mobile et des approches en sciences sociales mobilisées pour l'étudier. Le cadrage théorique qui articule l'analyse des artefacts et des formes innovantes de la médiation socio-technique aux stratégies socioéconomiques des acteurs sera ensuite précisé. En second lieu, les stratégies et les modèles socioéconomiques des acteurs industriels présents dans la téléphonie mobile seront étudiées en corrélation avec les conséquences des mutations observées dans les modes d'accès à l'information, notamment dans le domaine de la santé mobile. Nous ferons apparaître les modalités selon lesquelles de nouvelles formes éditoriales, centrées sur les nouveaux modes de production et de diffusion d'informations ou de connaissances, conditionnent la production et l'accès mobile à des contenus. Nous montrerons que ces modes d'accès sont le fruit des relations croisées entre des innovations techniques, des modèles socioéconomiques, des modes de régulation et des pratiques éditoriales.

\section{Un domaine de recherche interdisciplinaire, entre santé connectée et bien-être connecté}

La santé mobile peut être appréhendée comme un marché et une industrie [BUT 16] qui soulève des problématiques complexes en termes de sécurité de l'information [HAN 16] et de régulation juridiques liée aux modes d'utilisation et de protection des données [THI 16]. Elle constitue un domaine hybride regroupant différents types d'acteurs aux motivations hétérogènes: les pouvoirs publics y voient l'opportunité d'usages en prévention [CAM 16], d'une accessibilité améliorée des services de santé (par la télémédecine) et d'une réduction des dépenses en santé publique ; les professionnels de santé expérimentent la téléassistance mobile et le suivi médical à distance (notamment des maladies chroniques). De plus, la participation des publics à la construction des données de santé peut s'inscrire dans une démarche d'autonomisation (empowerment) et de démocratie sanitaire. De nombreux travaux internationaux récents, répertoriés par la Haute Autorité de la santé, portent sur l'évaluation d'applications en santé mobile pour smartphone menés selon une pluralité d'approches disciplinaires et thématiques, concernant notamment : les publics et les finalités (médecine préventive, suivi médical, téléassistance, observance, bien-être...), la sécurité et la gestion des données, les contenus de santé, les systèmes techniques, l'utilisation et l'usage [HAS $16: 8-13,56-59]$. Des études portent également sur les «mobinautes » (publics et professionnels de santé) usagers ou non usagers de ces applications mobiles, étudiés en termes de connaissances, de perceptions, d'usages, d'attentes et de besoins [CNO $15: 15-16]$. Les publics dépassent le domaine de la e-santé et de ses solutions médicalisées concernant le suivi des maladies chroniques, l'observance ou l'accompagnement des personnes dépendantes. Ils incluent des usagers souhaitant avoir une connaissance et une maitrise améliorées de leur santé et de leur bien-être. Des approches compréhensives s'intéressent au "soi quantifié » (quantified self) dans les «rituels d'automesure numérique » [ARR 13] et de la «mise en chiffres de soi » associée à une dimension de partage au sein de communautés [PHA 13].

L'écosystème informationnel numérique (sites web, forums, réseaux sociaux...) a déjà suscité des tensions résultant de l'ouverture de l'accès à l'information médicale et des modes de communication ouvrant à la participation des publics. Les applications de santé introduisent des problématiques spécifiques liées aux dispositifs socio-techniques et aux nouvelles modalités d'intermédiation, d'information ubiquitaire et de communication. 
3. «Objets-frontières», stratégies socio-économiques et formes innovantes de la médiation socio-technique dans la mobilité équipée

Les innovations, c'est-à-dire les longs processus sociaux par lesquels se diffusent des inventions dans leur parcours jusqu'aux usagers d'objets socio-techniques [ALT 00], sont nombreuses dans le domaine des industries de la communication. En ouvrant à l'accès à distance à Internet en situation de mobilité via des objets connectés, les artefacts électroniques ont progressivement évolué en interrelation avec les pratiques informationnelles et communicationnelles des publics.

Les innovations occupent une place centrale dans le domaine très concurrentiel des industries de la communication. La prolifération d'objets nomades et d'interfaces multiplie désormais les points d'entrée techniques et, par ce biais, les médiations à l'information. Le concept d'objet-frontière aide à appréhender ces objets techniques, hybrides et multitâches, qui combinent des fonctions de téléphonie, d'Internet mobile, de lecture multimédia, tout en donnant accès à l'information. Ce concept est évoqué en référence à des «objets, abstraits ou concrets, dont la structure est suffisamment commune à plusieurs mondes sociaux pour qu'elle assure un minimum d'identité au niveau de l'intersection tout en étant suffisamment souple pour s'adapter aux besoins et contraintes spécifiques de chacun de ces mondes » [TRO 09: 8)]. Dans la santé mobile, une grande diversité d' « objets-frontières » connectés (des smartphones, des pèse-personnes, des piluliers, des montres, des lunettes, des vêtements...) constituent des objets hybrides à la croisée de plusieurs univers.

La dimension sociale des technologies de communication amène à questionner la « médiation sociotechnique » [AKR 93 ; JOU 93 ; LAT 93] et l'appropriation sociale des objets connectés, analysées dans les dispositifs et les pratiques informationnelles de la vie privée ou professionnelle. Au-delà de la conformité à des normes et à des standards internationaux relatifs à "l'utilisabilité » (usability) des matériels et des applications, les formes de la médiation socio-technique font apparaître que les innovations (des fabricants de matériels et de logiciels), en se combinant aux nouvelles pratiques éditoriales (des offreurs de services et de contenus) et informationnelles (des usagers), contribuent à construire une culture médiatique et technique. Le " cadre socio-technique » [FLI 95] s'inscrit dans la réflexion sur l'articulation de dimensions techniques et sociales. L'artefact devient ainsi un «médiateur, un acteur social, un agent, un actif qui fait partie d'un "programme d'action" composé de relations sociales, de relations de pouvoir, de lois, de morale... ». [LAT 93 : 44-45]. Inscrits dans le paradigme de l'innovation et de la théorie de la traduction, les travaux de la sociologie de l'innovation montrent la dimension sociale de l'innovation technique et mettent à jour les interactions et les négociations - allant jusqu'à des affrontements - des divers acteurs, y compris des actants, qui y participent [CAL 89 ; LAT 89]. Une culture numérique se construit, à la croisée des mutations dans les pratiques éditoriales des éditeurs de logiciels et de services, des modèles socio-économiques des industriels de la communication, et des pratiques informationnelles des usagers des nouveaux outils et services proposés [JUA 15].

Dans le même mouvement, les techniques numériques, en modifiant les conditions d'accès aux contenus, ont déstabilisé les équilibres socio-économiques et les modes d'organisation dans la publication des informations et des connaissances dans le secteur de la santé. Désormais, les acteurs des industries de la communication et de l'Internet jouent un rôle d'importance croissante dans le domaine hybride de la santé mobile. A cet égard, l'intermédiation centre l'attention sur l'introduction de nouveaux acteurs, dans leur statut et le rôle qu'ils jouent dans la médiation aux informations et aux connaissances.

Dans leurs rapports avec les éditeurs de contenus, les fabricants de matériels et de logiciels élaborent des stratégies et des modèles socio-économiques; il en résulte une offre d'outils et de services proposés aux usagers. Nous montrerons de quelle manière les stratégies et les modèles socioéconomiques des industriels, en articulation avec l'intervention d'instances de régulation nationales et 
européennes, contribuent fortement à structurer les formatages logiciels et techniques ainsi que les régimes d'accès aux informations et aux services.

\section{Modes d'accès mobile aux services de santé : vers l'intermédiation ou la désintermédiation?}

La question de l'intermédiation se pose sur le marché de la santé mobile en plein développement du fait de la généralisation de l'accès mobile à Internet via des smartphones et des services en santé qui s'appuient sur la mobilité équipée. En premier lieu, l'évolution de l'internet fixe vers le développement de l'accès mobile conditionne de manière croissante l'accès à l'information. L'informatique mobile a investi ces marchés [MED 17] ${ }^{1}$. Le succès des smartphones a contribué à faire évoluer les usages, et la part des personnes se connectant plus fréquemment aux sites, portails et applications mobiles, augmente. Cette orientation va de pair avec l'accès aux télécommunications sans fil (communication WiFi et technologie Bluetooth, technologie iBeacon d'Apple), l'utilisation d'un système d'identification électronique (puces RFID) et l'Internet à haut débit. En second lieu, le marché de la santé mobile est en pleine croissance selon le rapport annuel de Research 2 Guidance, analyste des marchés d'applications mobiles : à la fin de l'année 2015, dans le monde entier, 7900 applications en santé mobile (mHealth apps) étaient disponibles (dont $55 \%$ en Europe et $28 \%$ aux Etats-Unis, $2 \%$ en Afrique, $2 \%$ en Amérique du Sud, 13\% dans les APAC), et 2600 praticiens y avaient recours. Ce marché en croissance de plus de 5 milliards de dollars devrait atteindre 26 milliards de dollars en 2017 et 31 milliards de dollars en 2020, essentiellement grâce aux services fournis en relation avec les applications [RES $16: 10]$. Il est majoritairement européen et nord-américain.

Dans ces reconfigurations industrielles, stratégiques et techniques de grande ampleur, de nouveaux acteurs de l'industrie informatique et de la communication se sont implantés - par l'intermédiaire de leurs produits et de leurs services, de leurs modèles socio-économiques, de leurs stratégies - sur le marché de la santé mobile. En 2015, 23\% des entreprises éditrices d'applications de santé mobile étaient des entreprises IT/TECH, 27\% des développeurs informatiques, $5 \%$ étaient spécialisés dans les services de télésanté. En regard, les acteurs historiques du secteur de la santé sont minoritaires sur le nouveau segment de la santé mobile (7\% dans les dispositifs médicaux, $6 \%$ dans le secteur pharmaceutique, $4 \%$ dans l'assurance santé, $4 \%$ d'hôpitaux) dans lequel les nouveaux entrants se sont implantés [RES $16: 1$ ].

Dans l'accès mobile, quels sont les liens entre les modèles socio-économiques et les choix techniques - matériels et logiciels - des acteurs industriels ? Quelles en sont les conséquences sur les régimes d'accès aux informations ? Dans ce domaine innovant, les aspects matériels et techniques sont prégnants dans l'accès à l'information pour l'usager. Les possibilités ouvertes par la mobilité équipée et l'expansion des réseaux de télécommunication pourraient laisser penser qu'ils facilitent largement la circulation des informations. Or les choix qui sont faits s'inscrivent, en exploitant la plasticité du processus informationnel, autant dans des stratégies d'ouverture de l'information que dans des stratégies de contrôle d'accès.

Sur un marché international, la concurrence est très vive entre les fabricants de matériels électroniques et informatiques, et de logiciels. Ces entreprises valorisent leurs innovations technologiques en association avec le marquage identitaire de leurs produits. Windows Mobile, iPhone $O S$ et Android sont les principaux systèmes d'exploitation pour mobiles qui se partagent le marché du smartphone. Safari d'Apple, Chrome d'Android, IE de Microsoft, sont les navigateurs Internet majeurs. Parmi les plus connus, les matériels de différentes marques (Samsung, Nokia, Acer, Sony Ericsson, LG, HTC, Motorola...) fonctionnent sous système d'exploitation Android (de Google) ou Windows Phone

\footnotetext{
${ }^{1}$ Médiamétrie, 15/02/2017, « L'Audience Internet Mobile en France en décembre 2016 ». 40\% de la population a visité au moins un site/une application marchand(e) généraliste depuis son mobile en décembre. En décembre 2016, 22,1 millions d'individus se sont rendus sur les sites et applications de la sous-catégorie « Sites marchands généralistes » depuis un mobile, soit 2 Français sur 5.
} 
(de Microsoft, avec une suite Office mobile). Ces acteurs testent également de manière opportuniste le marché des objets connectés portables de consommation courante, à l'instar de Nokia qui a racheté l'entreprise française Withings et propose un produit innovant de montre connectée reliée à l'application dédiée Withings Health Mate sur smartphone.

$\mathrm{Au}$ final, trois multinationales américaines (Apple, Google, Microsoft) occupent la majeure partie de ce marché informatique international, très concurrentiel et qui repose sur des technologies innovantes. Elles font partie des acteurs majeurs du web, souvent nommés les GAFA (Google, Apple, Facebook, Amazon) qui, avec Microsoft, représentent les cinq plus importantes capitalisations boursières et dont une partie de l'activité repose sur l'exploitation des données des usagers. Ces derniers sont contraints d'utiliser les matériels et les logiciels de la mobilité équipée afin d'accéder aux informations et aux services de santé mobile.

Quant aux éditeurs d'applications de santé mobile, les contenus étant associés à des logiciels, ils doivent se conformer aux normes édictées par ces acteurs dominants qui se sont imposés en nouveaux intermédiaires pour le développement et le téléchargement des applications. La publication multiplateforme sur Android et iOS est la norme pour les éditeurs de santé mobile, $75 \%$ d'entre eux produisant des applications pour les deux plateformes. Les répartitions se font, selon des usages non exclusifs des plateformes, entre Android (88\%), iOS (84\%), Windows Phone (16\%), HTML5 (29\%) [RES $16: 2]$.

Les grandes organisations du web et de la communication, à l'instar des «objets-frontières » qu'elles éditent, peuvent être caractérisées d' « organisation-frontière »; elles se situent à la croisée de plusieurs mondes industriels et d'activités tout en s'appuyant sur leur spécialisation informatique, sur Internet et sur les matériels électroniques. Sur un marché concurrentiel et instable, elles déploient des stratégies de développement différentes que leur mise en concurrence les amène à faire évoluer et à adapter en fonction des initiatives de leurs concurrents.

Selon une logique de concentration, la stratégie de la société Apple, en regard des pratiques de ses concurrents, illustre de quelle manière la maîtrise des matériels et des logiciels, dans l'accès mobile, lui permet de verrouiller l'accès à l'information et aux services tout en imposant l'achat de ses appareils. Ces contraintes s'exercent autant sur les développeurs d'applications pour mobile que sur les éditeurs d'applications et les usagers d'outils nomades. Cette logique de verrouillage est exercée en premier lieu envers les développeurs. Depuis 2010, avec l'arrivée de l'iPhone OS 4.0, Apple a modifié les conditions d'utilisation destinées aux développeurs en imposant une plateforme de développement et un langage d'écriture (Objective-C) nécessaires au fonctionnement des applications sur iOS et à leur autorisation de figurer sur le logiciel de vente d'applications (Apple Store). Cette situation est inédite dans l'histoire de l'informatique, au cours de laquelle aucun éditeur de système d'exploitation n'avait imposé jusqu'ici un langage d'écriture de logiciels. A l'inverse, pour conquérir le marché des ordinateurs personnels, la stratégie de Microsoft avait été de laisser aux développeurs la liberté d'écriture des applications, à la condition qu'elles soient uniquement exécutables sous Windows et non compatibles avec les autres systèmes d'exploitation. La stratégie d'Apple semble être de tenter d'empêcher qu'une société concurrente n'impose un standard multi-plateformes sur le marché mobile. La société tire parti de sa position dominante dans différents segments de marché sur des smartphones, notamment en e-santé, et du succès de sa tablette pour imposer son système d'exploitation dans ces segments.

Et comme les usagers qui voudront accéder aux applications d'Apple pour iPhone OS ou pouvoir utiliser celles déjà acquises devront (r)acheter un appareil Apple, les ventes de la société s'en trouveront augmentées d'autant. La société Apple se situe dans une forme particulière de convergence organisationnelle, l'intégration verticale, où l'entreprise maîtrise toutes les étapes de la filière de production par des verrous techniques. Selon une logique propriétaire, elle détient son pouvoir du fait 
qu'elle fabrique les matériels, développe le système d'exploitation, et développe ou contrôle l'accès aux applications par l'intermédiaire du logiciel Apple Store.

Google a fait un choix différent en jouant la carte du produit ouvert. Il s'agit d'une concentration horizontale avec un positionnement sur des marchés différents dans le secteur du logiciel, et plus récemment de l'accès mobile. Cet acteur industriel majeur est spécialisé dans le domaine du logiciel (à l'origine son moteur de recherche) et ne fabrique pas de tablette ni de téléphone portable. Il a pris place sur le marché des logiciels de l'internet mobile avec un système d'exploitation pour téléphone mobile (Android) qui est un logiciel libre et ouvert, non dépendant du téléphone. Ces caractéristiques techniques signifient que tous les fabricants de téléphones portables peuvent s'approprier le système Android et y développer des applications comme ils le souhaitent sur le plan technique. Le contrôle est exercé en aval au niveau du canal de diffusion imposé via la boutique en ligne Android market. Son navigateur pour mobile, Google Chrome, est le deuxième logiciel le plus utilisé sur les téléphones portables, et a dépassé, en termes de nombre d'utilisateurs, celui d'Apple (Safari).

L'analyse des stratégies de développement de ces « organisations-frontières » de l'Internet, de l'informatique et du web fait apparaître les modes de contrôle du marché de l'Internet mobile et de collecte de données qu'elles mettent en œuvre. Ils sont composés de plusieurs éléments clés à maîtriser: les matériels informatiques permettant l'accès mobile, les systèmes d'exploitation qui pilotent le mode de fonctionnement de ces matériels, les navigateurs web pour mobile pour l'accès à Internet, le système de diffusion d'applications qui ouvre leur accès (selon un modèle économique à définir), et les applications pour smartphone qui permettent au public d'accéder aux contenus. Le verrouillage des modes d'intermédiation électronique et d'interopérabilité sont centraux pour le contrôle de la diffusion des contenus et des services.

La place des opérateurs de services de communication, des assureurs et des professionnels de la santé reste à définir dans ce nouvel environnement. Des partenariats avec des acteurs de santé (hôpitaux, laboratoires pharmaceutiques...) visent à susciter des projets innovants en santé mobile et connectée dans lesquels ils soient partenaires. Les publics sont partie prenante dans des pratiques émergentes telles que "l'ubimédecine », « une pratique médicale basée sur la réception et l'analyse de données de santé recueillies à l'initiative de l'usager en des moments et des lieux multiples » en dehors des cadres médicaux habituels du bureau de consultation ou de la chambre d'hôpital [CNO 15:12]. Des inquiétudes se font jour concernant certains partenariats, notamment entre des sociétés d'assurance et des acteurs de l'Internet.

\section{Les formes de régulation de l'accès mobile : un questionnement sociopolitique}

L'accès mobile pose problème concernant la protection des données personnelles. Par l'intermédiaire des applications téléchargées sur les téléphones, des informations sur les usagers sont récupérées et sont vendues dans des finalités marketing à des régies publicitaires. Les données peuvent concerner la localisation de l'utilisateur du téléphone, l'identifiant unique du téléphone (dont la confidentialité n'est pas protégée) ou les données liées à son identité (âge, sexe, etc.).

Plusieurs types d'organisations interviennent dans l'élaboration de la règlementation sur la protection des données personnelles. Les textes incluent les actes législatifs publics et les modes de régulation secondaire constitués par les normes et les standards techniques ou de management, cet ensemble évoluant avec les innovations des technologies de l'information et de la communication (Juanals, 2014). Les pouvoirs publics et les organisations professionnelles du secteur de la santé diffusent les dispositions légales et règlementaires relatives à l'hébergement, au partage d'informations et aux traitements de données à caractère personnel «dès lors que les données traitées par l'Apps/OC sont relatives à une personne physique identifiée ou qui peut être identifiée, directement ou 
indirectement. [...] Les données de santé2, particulièrement sensibles font l'objet d'un encadrement renforcé. $»^{3}$ [HAS $\left.16: 12\right]$. Dans le domaine de la santé mobile, ainsi que le précise la Haute Autorité de Santé, la conception et l'exploitation des objets connectés et des données de santé doivent "se conformer aux cadres juridiques (national et européen) existants notamment en matière de dispositifs médicaux, d'échange d'informations et de traitement des données de santé à caractère personnel ». Les applications susceptibles d'être qualifiées de dispositif médical relèvent de l'article L. 5211-1 du code de la santé publique, complété par les précisions de l'agence nationale de sécurité du médicament et des produits de santé (ANSM). L'hébergement de données de santé à caractère personnel pour le compte de personnes physiques ou morales à l'origine de la production ou du recueil desdites données ou pour le compte du patient lui-même, doivent respecter l'article L. 1111-8 du Code de la santé publique [HAS $16: 13$ ). Au niveau européen, Le livre vert sur la santé mobile (Green paper on mobile Health, 2014) accompagne les pratiques médicales et de santé publique reposant sur des dispositifs mobiles.

Face à la prolifération et à l'hétérogénéité des produits en santé mobile, des instances publiques et des organisations professionnelles de santé publient, en complément des textes législatifs et règlementaires, des documents d'orientation sur la santé mobile à l'intention des professionnels de santé, des associations de patients et des éditeurs d'applications. Ces acteurs font part de leurs préoccupations concernant l'impact de ces nouveaux produits ou services sur la santé publique tout en contribuant à la compréhension des besoins et des attentes des usagers. Ils s'intéressent également à l'évolution des pratiques en direction d'une médecine personnalisée qui respecte les principes de sécurité de l'information et de protection des données personnelles. Ces documents sont des référentiels de bonnes pratiques [HAS 16], des livres blancs, des analyses en innovation et prospective [CNI 14] ou des enquêtes (menées depuis 2013) auprès des publics de la santé mobile [CNO 15]. Ils orientent les pratiques des professionnels de santé selon des principes de respect des règlementations, d'adoption de labels et de référentiels d'évaluation communs pour la profession, et de considérations éthiques. Il en est de même au niveau européen et international [HAS 16].

\section{Conclusion et ouvertures de recherche}

Les enjeux relatifs au développement de la production et de l'accès mobile à l'information dans le domaine de la santé mobile revêtent des aspects à la fois d'ordre sanitaire, industriel, économique et technique ; ils ont également des répercussions sociales et politiques quant à la démocratie sanitaire et à la protection des données personnelles sur des outils nomades. Ils ouvrent à un débat et à des travaux portant sur l'accès mobile, dans ses relations avec la communication et la circulation d'informations et de connaissances en santé. En mettant en relation la matérialité des dispositifs socio-techniques avec des modes de production, de communication, de participation, et de circulation de ressources en santé qu'elle est susceptible de favoriser dans l'espace public, il apparaît que le choix d'une logique d'ouverture, en articulation avec un choix de modèle socio-économique, est en relation avec des standards et des formats ouverts, interopérables et prenant en compte le respect de la vie privée. Ces dispositifs pourraient être porteurs d'autres priorités et d'idéologies différentes, voire divergentes, qui utiliseraient des moyens similaires. C'est pourquoi il est important de discuter, de manière critique, de leurs caractéristiques, des acteurs qui les portent et des rapports de force qui s'instaurent, des modèles socio-économiques sous-jacents et de leurs conséquences.

\footnotetext{
${ }^{2}$ « Les données à caractère personnel relatives à la santé physique ou mentale d’une personne physique, y compris la prestation de services de soins de santé, qui révèlent des informations sur l'état de santé de cette personne » (règlement européen du 27 avril 2016).
}

\footnotetext{
${ }^{3}$ Loi n ${ }^{\circ}$ 78-17 du 6 janvier 1978 relative à l'informatique, aux fichiers et aux libertés ; - Règlement (UE) 2016/679 du Parlement européen du 27 avril 2016.
} 


\section{Bibliographie}

[AKR 93] AKRICK M., « Les formes de la médiation technique », Réseaux n 60, CNET.

[ALT 00] ALTER N., L’innovation ordinaire, Paris, PUF, 2000.

[ARR 13] ARRUABARRENA B., QUETTIER P., «Des rituels de l'automesure numérique à la fabrique autopoiétique de soi », Les Cahiers du numérique 2013/3 (Vol. 9), p. 41-62, 2013.

[BEN 09] BENGHOZI J.-P., BUREAU S., MASSIT FOLLEA F., L'Internet des objets. Quels enjeux pour l'Europe ?, Éditions de la Maison des sciences de l'homme, Paris, 2009.

[BUT 16] ButhiOn A. "Marché unique européen du numérique et politique française de santé », Annales des MinesRéalités industrielles 2016-3 (Août 2016), p. 61-65, 2016.

[CAL 86] CALLON M. (1986), «Eléments pour une sociologie de la traduction, la domestication des coquilles St-Jacques et des marins pêcheurs dans la baie de St-Brieuc », in L'Année Sociologique, $n^{\circ}$ spécial « La sociologie des sciences et des techniques », vol. 36, p. 169-208, 1986.

[CAM 16] CAMBON L., «Objets connectés, mobiles, communicants en prévention: dépasser l'outil, penser l'intervention », Santé Publique 2016/1 (Vol. 28), p. 5-6, 2016.

[GRE 66] GREENFIELD A., Everyware. The Dawning Age of Ubiquitous Computing, New Riders, 2006.

[HAN 16] Hantouche C., «Peut-on sécuriser l’Internet des Objets ? », Sécurité et stratégie 2016/2 (22), p. 31-38, 2016.

[JOU 93] JOUET J (1993), « Pratiques de communication : figures de la médiation », Réseaux n 60, CNET, 1993.

[JUA 09] JUANALS B., «La traçabilité dans les systèmes d'information : un questionnement politique sur la gouvernance des populations », Communications \& Langages, n 160, pp. 49-61, 2009.

[JUA 14] JuANALS B., «Protection des données personnelles et TIC au cœur des enjeux de société et de la mondialisation: les mécanismes d'un contrôle distribué», p. 228-253, TIC\&Société, volume 8 (1-2), 1er et 2e semestres 2014, <http://ticetsociete.revues.org/1475>, 2014.

[JUA 15] JUANALS B., "TIC en société : pour une approche info-communicationnelle de la culture numérique », dans MASSElOt C., RASSE P. (dir.), Sciences, techniques et société. Recherches sur les technologies digitales, Paris, L'Harmattan-SFSIC, p. 25-34, 2015.

[JUA 10] JuAnals B Noyer J.-M. (coord.), Technologies de l'information et intelligences collectives, Paris, Hermès Science Publications-Lavoisier, 2010.

[LAT 93] Latour B., Petites leçons de sociologie des sciences, "La clé de Berlin », p. 33-46, La Découverte, Paris, 1993.

[LAT 89] LATOUR B., La science en action, La Découverte, Paris (1re éd. ang. 1987), 1989.

[TRO 09] TROMPETTE P., VINCK D., « Retour sur la notion d'objet-frontière », Revue d'anthropologie des connaissances, 2009/1 - Vol. 3, n 1, p. 5-27, 2009.

[PHA 13] PHARABOD A-S., NiKOlSKI V., GRANJON F., " La mise en chiffres de soi: Une approche compréhensive des mesures personnelles ». Réseaux 177, no 1 (2013): 97, 2013.

[THI 16] THIERACHE C., «L'agrégation des données ouvertes dans le cadre de plateformes : les objets connectés dans le domaine de la santé », LEGICOM 2016/1 (N 56), p. 101-109, 2016.

\section{Rapports cités}

[CNI 14] CNIL (2014), « Le corps, nouvel objet connecté », in Cahiers IP. Innovation et prospective, n 2, CNIL. https://www.cnil.fr/sites/default/files/typo/document/CNIL_CAHIERS_IP2_WEB.pdf

[CN0 15] Conseil national de l'ordre des médecins (2015), Santé connectée. De la e-santé à la santé connectée. Livre blanc, Paris, CNOM. www.conseil-national.medecin.fr/sites/default/files/medecins-sante-connectee.pdf

[CEU 14] Commission européenne (avril 2014), Livre vert sur la santé mobile.

[HAS 16] Haute Autorité de Santé (octobre 2016), Référentiel de bonnes pratiques sur les applications et les objets connectés en santé (Mobile Health ou mHealth). www.has-sante.fr 
[MED 17] Médiamétrie (15/02/2017), « L’Audience Internet Mobile en France en décembre 2016 ».

http://www.mediametrie.fr/internet/communiques/l-audience-internet-mobile-en-france-en-decembre-2016.php?id=1621

[WHO 11] World Health Organization (2011), mHealth - New horizons for health through mobile technologies, Global Observatory for eHealth series, Volume 3, Geneva: WHO; 2011. www.who.int/goe/publications/goe_mhealth_web.pdf

[RES 16] Research 2 Guidance (octobre 2016), «mHealth App Developer. Economics 2016. The current status and trends on the mHealth app market, 6th annual study on mHealth app publishing passed on 2,600 plus respondents », http://research2guidance.com/ 